\title{
Regularity, Asymptotic Solutions and Travelling Waves analysis in a porous medium system to model the interaction between invasive and invaded species
}

\author{
José Díaz ${ }^{1}$ and Antonio Naranjo ${ }^{1}$ \\ ${ }^{1}$ Francisco de Vitoria University
}

April 2, 2021

\begin{abstract}
This work provides an analytical approach to characterize and determine solutions to a porous medium system of equations with views in applications to invasive-invaded biological dynamics. Firstly, the existence and uniqueness of solutions are proved. Afterwards, profiles of solutions are obtained making use of the selfsimilar structure that permits to show the existence of a diffusive front. The solutions are then studied within the Travelling Waves (TW) domain showing the existence of potential and exponential profiles in the stable connection that converges to the stationary solutions in which the invasive specie predominates. The TW profiles are shown to exist based on the geometry perturbation theory together with an analytical-topological argument in the phase plane. The finding of an exponential decaying rate (related with the advection and diffusion parameters) in the invaded specie TW is not trivial in the non-linear diffusion case and reflects the existence of a TW trajectory governed by the invaded specie runaway (in the direction of the advection) and the diffusion (acting along a finite speed front or support).
\end{abstract}

\section{Hosted file}

Travelling_Waves_analysis_in_a_porous_medium_system_of_equations_with_coupled_reaction_and_advection (1 available at https://authorea.com/users/405462/articles/516427-regularity-asymptoticsolutions-and-travelling-waves-analysis-in-a-porous-medium-system-to-model-theinteraction-between-invasive-and-invaded-species 